\title{
Nuevos registros de poblaciones amenazadas de Pilgerodendron uviferum D.Don (Florin) en su límite norte en la Cordillera de la Costa chilena
}

\section{New records of threatened Pilgerodendron uviferum D.Don (Florin) in their northern limit in chilean Coast Range}

\author{
Daniel P. Soto ${ }^{1}$, Jan R. Bannister ${ }^{2}$, Andrea I. Ríos ${ }^{3}$ \& Carlos Le Quesne ${ }^{1}$ \\ ${ }^{1}$ Instituto de Silvicultura y ${ }^{3}$ Laboratorio de Geomática, Universidad Austral, Casilla 567, Valdivia, Chile. \\ ${ }^{2}$ Waldbau Institut, Albert-Ludwigs Universität Freiburg, Tennenbacherstr. 4, 79108 Freiburg, Alemania. \\ danielsoto@uach.cl
}

\begin{abstract}
We describe twelve previously undocumented Pigerodendron uviferum D.Don (Florin) populations outside of protected areas at the northern limit of the Chilean Coast Range (from $39^{\circ} 43^{\prime}-40^{\circ} \mathrm{S}$ ), extending their northern limits. These small swamp-populations are isolated within a matrix of pastures and exotic forest plantations, and are subject to "chronic" anthropogenic disturbances.
\end{abstract}

Pilgerodendron uviferum (D.Don.) Florin es una conífera (Cupressaceae) endémica, dioica y longeva de los bosques Templados Sudamericanos, que habita preferentemente zonas frías y con abundante precipitación $(>3.000 \mathrm{~mm})$ y ocupa terrenos planos con drenaje deficitario (Lara et al. 2006, Soto \& Figueroa 2008). Su rango de distribución abarca unos $1.600 \mathrm{~km}\left(39^{\circ} 35^{\prime} \mathrm{S}\right.$, Martínez 1981; 55 $30^{\prime} \mathrm{S}$, Pisano 1977), lo que la cataloga como la conífera más austral del mundo (Lara et al. 2006). Una de las particularidades de esta especie es la gran durabilidad y excelente calidad de su madera, condición que ha impulsado su masiva explotación desde el siglo XVII hasta la actualidad (Martínez \& Muñoz 1988, Lara et al. 2006). Debido a esto, es muy común encontrar bosques de $P$. uviferum con gran parte de sus árboles muertos en pie a causa de incendios que han modificado la estructura y composición de estos bosques (Lara et al. 2006, Soto \& Figueroa 2008, Bannister et al. 2008). Esta constante presión ha producido un enorme deterioro para la especie a lo largo de su distribución y por esta razón ha sido catalogada como "Vulnerable" (Walter \& Gillett 1998, Farjon \& Page 1999) por la IUCN e incluida en el Apéndice 1 de la convención CITES (Hechenleitner et al. 2005).

En la actualidad, se desconoce con exactitud cuánta superficie de bosques con $P$. uviferum ha sido afectada por alteraciones antrópicas (ej. fuego y cortas de extracción), sin embargo, existe el consenso que son pocos los rodales inalterados (Lara et al. 2006). Esta dramática situación se acentúa en el límite norte de la Cordillera de la Costa, donde recientemente se detectó la presencia de poblaciones marginales dispersas, de escasa superficie $(<$ 2 ha) incluidas en una matriz de plantaciones intensivas de Pinus radiata D.Don, especies del género Eucalyptus y/o usos agro-ganaderos (Soto et al. 2007), las cuales por su pequeño tamaño no fueron incluidas en el Catastro y Evaluación de Recursos Vegetacionales Nativos de Chile (CONAF et al. 1997). Bajo este escenario, se propuso registrar, desde el año 2002 a la fecha, la mayor cantidad posible de sitios indocumentados con presencia de $P$. uviferum, fuera del SNASPE, en la Cordillera de la Costa al norte y oriente de la Reserva Forestal Valdivia, donde actualmente la zona estudiada es afectada por una fuerte transformación del uso del suelo (Bannister et al. 2009). Estas amenazas sobre la flora nativa le dan una mayor importancia a estas poblaciones, ya que biogeográficamente P. uviferum ha registrado las mayores diferencias genéticas interpoblacionales en el área norte de su distribución (Allnutt et al. 2003). De esta manera, estos nuevos registros podrían aportar al mejor entendimiento biogeográfico de la especie, justo donde se ha documentado que poblaciones norteñas serían remanentes de flora del último periodo glacial (Premoli et al. 2002).

Después de 8 años de prospecciones colaborativas, en las cuales se recorrió la zona buscando rodales de $P$. uviferum basado en información proveniente de pequeños propietarios de bosques, este estudio entrega el registro de 12 nuevas poblaciones ubicadas en la Región de Los Ríos (Tabla I). La estructura y edad de las poblaciones no fue estudiada, sin embargo es similar a la estructura de las poblaciones estudiadas con anterioridad por Soto et al. 
Nuevas poblaciones de Pilgerodendron uviferum en su límite norte: SoTo, D.P. ET AL.

(2007). Las poblaciones de "Las Minas" (3943`14'S y $\left.73^{\circ} 21^{\prime} 00^{\prime \prime O}\right)$ y "Punucapa" (3943`24”S y $73^{\circ} 17^{\prime} 33^{\prime}$ 'O) descrita en este estudio se constituirían en sus nuevos límites norte de $P$. uviferum en la Cordillera de la Costa, extendiendo el descrito por Soto et al. (2007), en Chancoyán $\left(39^{\circ} 45^{`} \mathrm{~S}\right)$. Los materiales de referencia se encuentran depositados en el herbario de la Universidad de Concepción (CONC)

La mayoría de estas poblaciones presentan severas alteraciones antrópicas que alteran su estructura $\mathrm{y}$ composición, y que pueden afectar su viabilidad y resiliencia. Dentro de las agravantes encontradas se destacan las plantaciones forestales exóticas, que con sus métodos tradicionales de cosecha a tala rasa y posterior repoblamiento masivo provocan una alteración en la disponibilidad de agua en el suelo (Vertessi 1999), la sustitución de la matriz vegetacional nativa (lo que forma fragmentos aislados de P. uviferum dentro de otros usos), la construcción de caminos, obras de drenaje, herbivoría y pisoteo animal, uso del fuego, tala de árboles (Tabla II). Dada esta crítica situación y de no mediar medidas urgentes de conservación y restauración, se estima que ocurriría la extinción local de algunas poblaciones en el mediano plazo, lo que resultaría en una pérdida patrimonial irreparable, teniendo en cuenta su condición de enclaves únicos de flora en su límite norte, en un paisaje altamente alterado (Fig. 1). Por tal motivo se recomiendan las siguientes medidas para la conservación de las poblaciones estudiadas: a) Mapeo exhaustivo de todos los rodales de $P$. uviferum que se encuentran en la costa de la Provincia de Valdivia, b) Exclusión de ganado de los rodales de $P$. uviferum rodeados por praderas, cultivos o áreas ganaderas mediante cercos, c) Prohibición de talas rasas en plantaciones exóticas cercanas a rodales de $P$. uviferum, d) Creación de áreas buffer entre plantaciones exóticas y rodales amenazados y restauración del bosque nativo ubicado dentro de ellas (Fig. 2).

TABLA I. Identificación, localización y material estudiado de las nuevas poblaciones de P. uviferum, todas localizadas en la provincia de Valdivia en la Región de Los Ríos, Chile.

TABLE I. Identification, location and study material of the new populations of P. uviferum in the Valdivia province of the Los Rios Region.

\begin{tabular}{|c|c|c|c|c|}
\hline PoBlación & Comuna & $\begin{array}{l}\text { Altitud } \\
\text { (m s.n.m.) }\end{array}$ & CoORdenadas & FECHA \\
\hline Las Minas & Valdivia & 300 & $39^{\circ} 43^{\prime} \mathrm{S} ; 73^{\circ} 20^{\prime} \mathrm{O}$ & $16-V-2009$ \\
\hline Punucapa & Valdivia & 85 & $39^{\circ} 43^{\prime} \mathrm{S} ; 73^{\circ} 17^{\prime} \mathrm{O}$ & $*$ \\
\hline Cuesta Soto & Valdivia & 322 & $39^{\circ} 48^{\prime} \mathrm{S} ; 73^{\circ} 08^{\prime} \mathrm{O}$ & $16-V-2009$ \\
\hline Estero Pilgüa & Corral & 25 & $39^{\circ} 53^{\prime} \mathrm{S} ; 73^{\circ} 19^{\prime} \mathrm{O}$ & 23-IX-2009 \\
\hline Angelitos & Corral & 250 & $39^{\circ} 54^{\prime} \mathrm{S} ; 73^{\circ} 17^{\prime} \mathrm{O}$ & 23-IX-2009 \\
\hline Camino viejo a La Unión, km 4 & Valdivia & 25 & $39^{\circ} 57^{\prime} \mathrm{S} ; 73^{\circ} 07^{\prime} \mathrm{O}$ & $16-V-2009$ \\
\hline Escuela Huiro & Corral & 60 & $39^{\circ} 57^{\prime} \mathrm{S} ; 73^{\circ} 39^{\prime} \mathrm{O}$ & 23-II-2009 \\
\hline Camino viejo a La Unión, km 9 & Valdivia & 49 & $39^{\circ} 58^{\prime} \mathrm{S} ; 73^{\circ} 07^{\prime} \mathrm{O}$ & $16-V-2009$ \\
\hline Huiro & Corral & 70 & $39^{\circ} 58^{\prime} \mathrm{S} ; 73^{\circ} 38^{\prime} \mathrm{O}$ & $*$ \\
\hline Playa Pelche & Corral & 42 & $39^{\circ} 59^{\prime} \mathrm{S} ; 73^{\circ} 40^{\prime} \mathrm{O}$ & 23-II-2009 \\
\hline Punta Galera & Corral & 80 & $39^{\circ} 59^{\prime} \mathrm{S} ; 73^{\circ} 40^{\prime} \mathrm{O}$ & 23-II-2009 \\
\hline Colún Norte & Corral & 30 & $40^{\circ} 00^{\prime} \mathrm{S} ; 73^{\circ} 40^{\prime} \mathrm{O}$ & 23-II-2009 \\
\hline
\end{tabular}

* Sin información debido a restricción de ingreso / Populations without floristic samples by access problems. 
Gayana Bot. 67(1), 2010

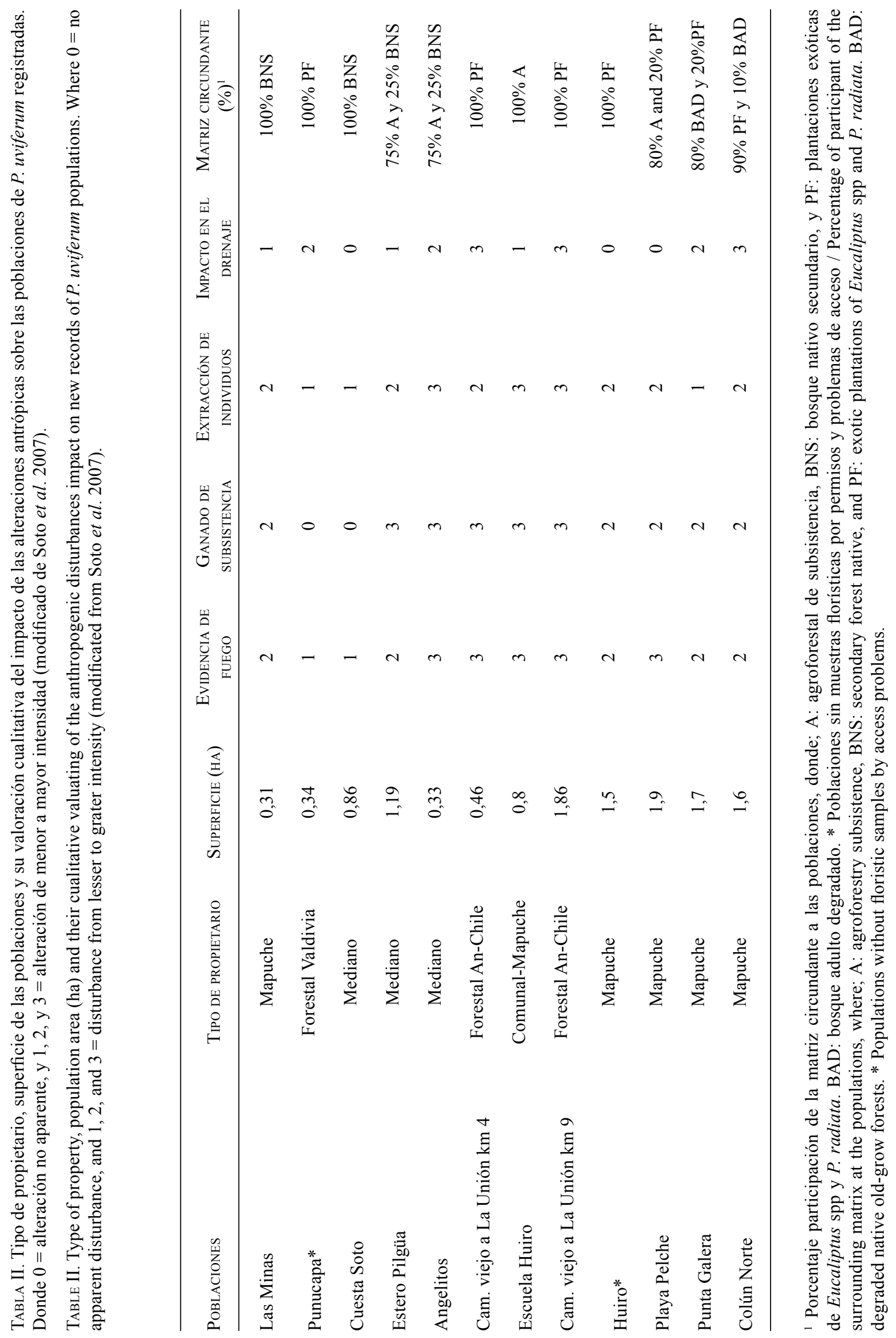



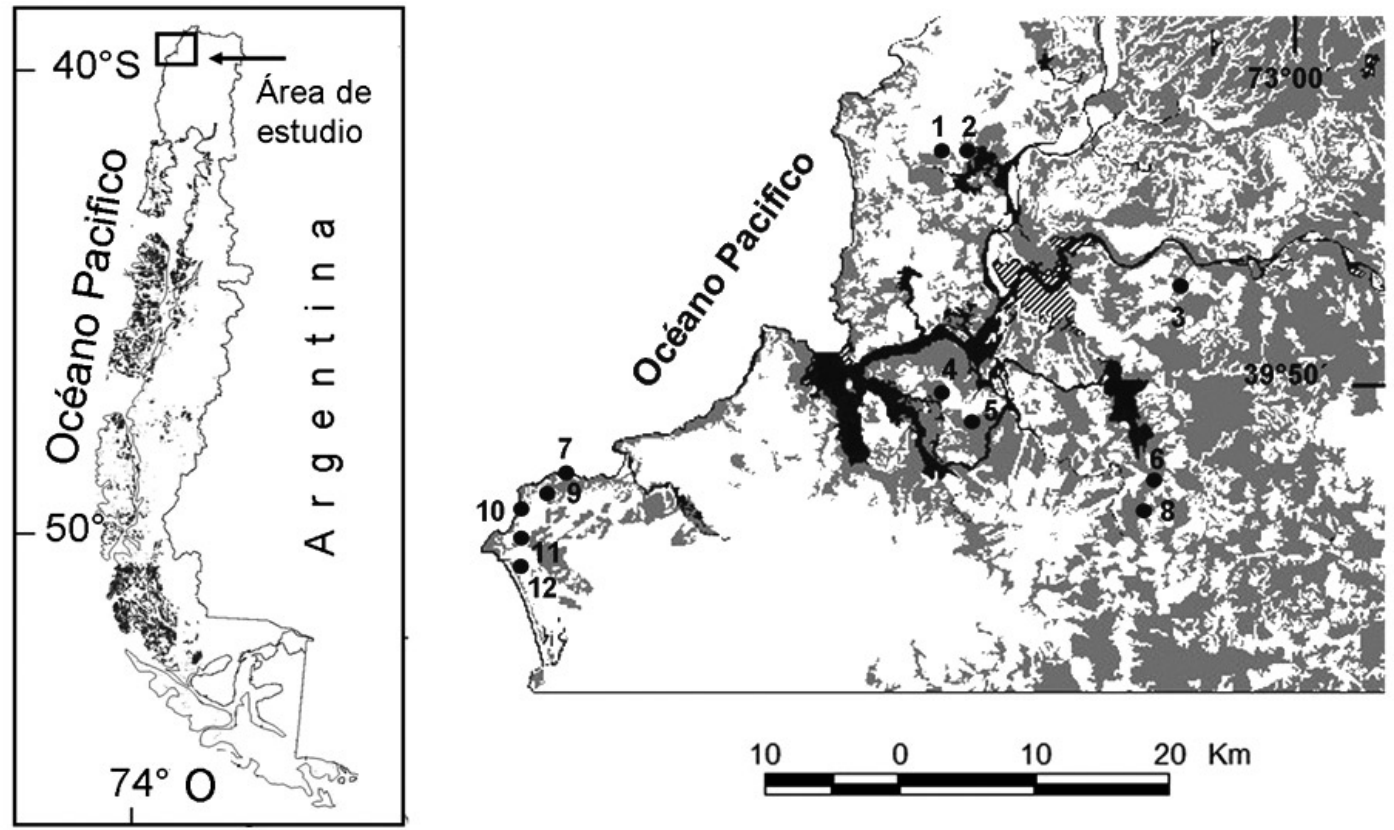

Figura 1. Ubicación de las nuevas poblaciones de P. uviferum en su limite norte de la Cordillera de la Costa. 1. Las Minas, 2. Punucapa*, 3. Cuesta Soto, 4. Estero Pilgüa, 5. Angelitos, 6. Camino viejo a la Unión Km 4, 7. Escuela Huiro, 8. Camino viejo a la Unión Km 9, 9. Huiro*, 10. Playa Pelche, 11. Punta Galera, 12. Colún Norte. En la figura se muestra en gris las plantaciones exóticas (Eucalyptus spp. y Pinus radiata) y los campos agrícolas, y en negro los ríos del área de estudiada. El área achurada muestra la ciudad de Valdivia.* poblaciones sin muestras florísticas por problemas de acceso. La figura del detalle muestra la distribución natural de $P$. uviferum en Chile.

FIgURE 1. Location of the new P. uviferum populations in their northern limit of Coast Range of Chile. 1. Las Minas, 2 . Punucapa*, 3. Cuesta Soto, 4. Estero Pilgüa, 5. Angelitos, 6. Camino viejo a la Unión Km 4, 7. Escuela Huiro, 8. Camino viejo a la Unión Km 9, 9. Huiro*, 10. Playa Pelche, 11. Punta Galera, 12. Colún Norte. Figure shows in grey the exotic tree plantations (Eucalyptus spp. and Pinus radiata) and farm lands, and black shows rivers in the study area. The dashed area show Valdivia City. * populations without floristic samples by access problems. The detailed figure shows the distributional range of $P$. uviferum in Chile.

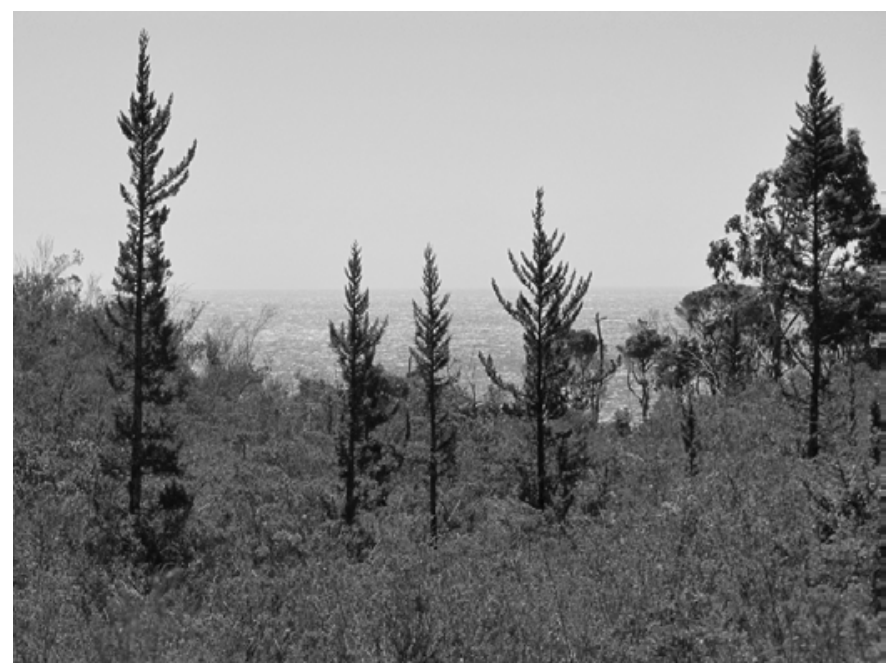

Figura 2. Fotografía de la población de P. uviferum en "Escuela Huiro", comuna de Corral, cercano al Océano Pacífico (Foto: Jan Bannister).

Figure 2. Photograph of the P. uviferum population in "Escuela Huiro", "Comuna de Corral", closed to the Pacific Ocean (Photograph: Jan Bannister). 


\section{AGRADECIMIENTOS}

A todos los colonos de las comunas de Valdivia y Corral por sus valiosas comunicaciones. A la Agrupación de Ingenieros Forestales por el Bosque Nativo (AIFBN) por su apoyo logístico, y a German Clasing de CONAF.

\section{BIBLIOGRAFÍA}

Allnutt, T.R., A.C. Newton, A.C. Premoli \& A. Lara. 2003. Genetic variation in the threatened South American conifer Pilgerodendron uviferum (Cupressaseae), detected using RAPD markers. Biological Conservation 100(1): 9-18.

Bannister, J.R., C. Le Quesne. \& A. Lara. 2008. Estructura y dinámica de bosques de Pilgerodendron uviferum afectados por incendios en la Cordillera de la Costa de la Isla Grande de Chiloé. Bosque 29(1): 33-43.

Bannister, J.R., P. Carcamo, I. Medel, J. Arias 2009. Caracterización de las Fuentes de Agua de la Comunidad Rural de Tres Chiflones, Provincia de Valdivia. Bosque Nativo 44: 3-6.

CONAF, CONAMA, BIRF, Universidad Austral de Chile, Pontificia Universidad Católica de Chile, Universidad Católica de Temuco. 1997. Catastro y Evaluación de los Recursos Vegetacionales Nativos de Chile. Informe Nacional con Variables Ambientales. Santiago, Chile. 88 pp.

Farjon, A. \& C.N. Page. 1999. Conifers. Status Survey and Conservation Action Plan. IUCN/SSC Conifer Specialist Group. IUCN, Gland, Switzerland and Cambridge, UK. $121 \mathrm{pp}$.

Hechenleitner, P., M.F. Gardner, P.I. Thomas, C. Echeverría, B. Escobar, P. Brownless \& C. Martínez. 2005. Plantas amenazadas del Centro-Sur de Chile: Distribución,
Conservación y Propagación. Universidad Austral de ChileRoyal Botanic Garden Edinburgh, Valdivia, Chile. 188 pp.

Lara, A., C. Donoso, B. Escobar, A. Rovere, A. Premoli, D.P. Soto \& J. Bannister. 2006. Pilgerodendron uviferum (D. Don) Florin. En: Autoecología de las especies arbóreas de los bosques templados de Chile y Argentina (ed C. Donoso), pp. 82-91. Marisa Cuneo Ediciones, Valdivia, Chile.

MartíneZ, O. 1981. Flora y fitosociología de un relicto de Pilgerodendron uviferum (D. Don) Florin, en el Fundo San Pablo de Tregua (Valdivia-Chile). Bosque 4(1): 3-11.

Martínez, O. \&. A.M. Muñoz. 1988. Aspectos conservativos de las coníferas chilenas. Bosque 9(2): 77-82.

Pisano, E. 1977. Fitogeografía de Fuego-Patagonia Chilena. I Comunidades vegetales entre las latitudes $52^{\circ}$ y $56^{\circ} \mathrm{S}$. Anales Instituto de la Patagonia 8: 121-250.

Premoli, A.C., C. Souto, A. Rovere, T.T. Allnutt \& A.C. Newton. 2002. Patterns of isozyme variation as indicators of biogeographic history in Pilgerodendron uviferum (D. Don) Florin. Diversity \& Distributions 8: 57-66.

Soto, D.P. \& H. FigueroA. 2008. Efectos de las alteraciones antrópicas sobre la estructura y composición de rodales de Pilgerodendron uviferum en la Cordillera de la Costa de Chile. Ecología Austral 18(1): 13-25.

Soto, D.P., C. Le Quesne, A. Lara \& M.F. Gardner. 2007. Precarious conservation status of Pilgerodendron uviferum forests in their northern distribution in the Chilean Coastal Range. Bosque 28(3): 263-270.

VERTESSY, R.A. 1999. The impact of forestry on streamflows: a review. In: J. Croke \& P. Lane (eds.), Forest Management for the protection of water quality and quantity. Cooperative Research Centre for Hydrology, report 99/6. pp. 93-109.

Walter, K.S. \& H.J. Gillett. 1998. 1997 IUCN Red List of Threatened Plants. IUCN, Gland, Switzerland and Cambridge, UK. 862 pp. 